\title{
Automatic Segmentation of Left Ventricular Myocardium by Deep Convolutional and De-convolutional Neural Networks
}

\author{
XL Yang, L Gobeawan, SY Yeo, WT Tang, ZZ Wu, Y Su \\ Institute of High Performance Computing, A*STAR, Singapore
}

\begin{abstract}
Deep learning has been integrated into several existing left ventricle ( $L V$ ) endocardium segmentation methods to yield impressive accuracy improvements. However, challenges remain for segmentation of $L V$ epicardium due to its fuzzier appearance and complications from the right ventricular insertion points. Segmenting the myocardium collectively (i.e., endocardium and epicardium together) confers the potential for better segmentation results.

In this work, we develop a computational platform based on deep learning to segment the whole $L V$ myocardium simultaneously from a cardiac magnetic resonance (CMR) image. The deep convolutional network is constructed using Caffe platform, which consists of 6 convolutional layers, 2 pooling layers, and 1 deconvolutional layer. A preliminary result with Dice metric of $0.75 \pm 0.04$ is reported on York MR dataset. While in its current form, our proposed one-step deep learning method cannot compete with state-of-art myocardium segmentation methods, it delivers promising first pass segmentation results.
\end{abstract}

\section{Introduction}

Automatic left ventricle (LV) segmentation from cardiac magnetic resonance (CMR) images is a challenging task and it is difficult to achieve highly accurate results without manual intervention. Over the years, various image processing techniques have been proposed to automatically and robustly segment the LV endocardium.

While intuition might suggest that segmenting the myocardium as a whole is more amenable from the perspective of human perception, it is non-trivial from an algorithmic point of view. Efforts to get a computer machine to learn to segment the images in a way similar to the human perception have recently been made possible by the increasingly powerful computing resources and the availability of vast amount of data.

Deep learning $[1,2,3]$ is an advanced machine learning technique that mimics the cognitive processing of the human brain using neural networks with multiple hidden layers. Among its many applications, this technique has been used in object recognition, and has been employed to segment the $\mathrm{LV}$ endocardium to yield impressive accuracy improvements $[4,5,6]$. However, challenges remain for segmenting the LV epicardium due to its fuzzier appearance. In addition, the epicardial boundary is not completely distinct due to the presence of the right ventricle insertion points.

On the other hand, past research works on LV myocardium (i.e., endocardium and epicardium) segmentation have been primarily confined to non-deep learning approaches. The results of these works are often compared in terms of a dice metric (DM), which is a statistic metric to measure the overlap between two superimposed image areas, in order to compare the accuracy of automatic segmentation result with that of the manual one.

$\mathrm{Hu}$ et al. proposed an automatic LV myocardium segmentation using local binary fitting and dynamic programming [7], producing a DM result of $0.89 \pm 0.04$ and $0.93 \pm 0.02$ for the endocardium and epicardium segmentation, respectively. Uzunbas et al. performed a semi-automatic LV myocardium segmentation using graph cut and deformable models [8]. They obtained a DM of $0.82 \pm 0.06$ and $0.91 \pm 0.03$ for the endocardium and epicardium segmentation respectively, using the MICCAI09 LV 2009 Challenge dataset [9]. Using a priori anatomical information, Sjögren et al. proposed a semi-automatic segmentation of MaR (Myocardium at Risk) [10] and obtained a DM of $0.85 \pm 0.07$ for some specific datasets. Similarly, the work by Tufvesson et al. was based on a priori regional criteria and infarct region from late gadolinium enhanced (LGE) CMR to define MaR using myocardial intensity classification by Expectation Maximization [11]. They obtained a DM of $0.85 \pm 0.08$ for 183 datasets. Petitjean and Dacher presented a thorough survey of other existing techniques up to year 2011 for related myocardium segmentation work [12].

Given the excellent results of machine learning (especially the recent deep learning) techniques for the LV endocardium segmentation, we explore the potential of deep learning for LV myocardium segmentation. 


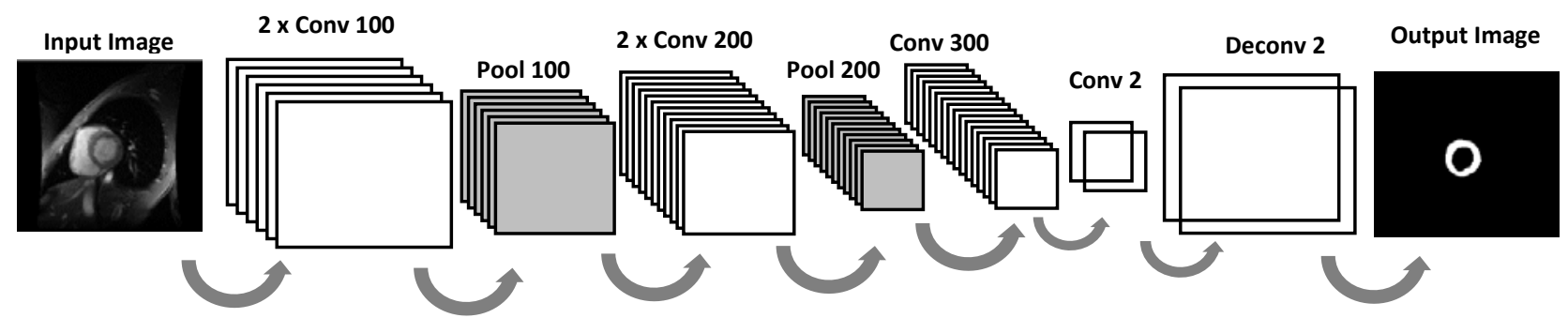

Figure 1. Layout of the designed deep learning model

Table 1. Layer parameters of the proposed model

\begin{tabular}{|l|l|l|l|l|}
\hline Layer & Depth & Filter & Stride & Parameters \\
\hline Input & 1 & --- & --- & 0 \\
\hline 1. Conv & 100 & $3 \times 3$ & $1 \times 1$ & $3 \times 3 \times 3 \times 100$ \\
\hline 2. Conv & 100 & $3 \times 3$ & $1 \times 1$ & $3 \times 3 \times 100 \times 100$ \\
\hline 3. Max & 100 & $2 \times 2$ & $2 \times 2$ & 0 \\
\hline 4. Conv & 200 & $3 \times 3$ & $1 \times 1$ & $3 \times 3 \times 100 \times 200$ \\
\hline 5. Conv & 200 & $3 \times 3$ & $1 \times 1$ & $3 \times 3 \times 200 \times 200$ \\
\hline 6. Max & 200 & $2 \times 2$ & $2 \times 2$ & 0 \\
\hline 7. Conv & 300 & $3 \times 3$ & $1 \times 1$ & $3 \times 3 \times 200 \times 300$ \\
\hline 8. Conv & 2 & $3 \times 3$ & $1 \times 1$ & $3 \times 3 \times 300 \times 2$ \\
\hline 9. DeConv & 2 & $3 \times 3$ & $4 \times 4$ & $3 \times 3 \times 2 \times 2$ \\
\hline Output & 1 & ---- & ---- & 0 \\
\hline
\end{tabular}

\section{Methodology}

In the past years, deep learning techniques, especially deep convolutional networks (DCN) [13], have outperformed the state-of-art methods in many computer vision tasks $[14,15,16]$. The typical use of convolutional networks is for categorizing images into different classes. The latest development of the fully convolutional networks (FCN) $[17,18]$ enables image segmentation by assigning a class label for each pixel within the image in a more elegant architecture, where the fully connected layers are replaced by spatial convolutional layers to learn per-pixel labels end-to-end from whole-image inputs and their corresponding whole-image ground truths. The method proposed in this study is developed based on the FCN architecture.

\subsection{Network design}

The deep convolutional network is constructed using the Caffe deep learning platform. It consists of 6 convolutional layers, 2 pooling layers, and 1 deconvolutional layer. The layout of the network architecture is shown in Figure 1. Each convolutional layer is followed by a Rectified Linear Unit (ReLU) activation function, while the de-convolutional layer is followed by a Softmax activation function. The layer parameters are trained using myocardium-annotated CMR datasets from York University [19]. The details of layer parameters in each layer are listed in Table 1.

\subsection{Accuracy function}

From the perspective of classification, myocardium segmentation from a given CMR image can be regarded as a binary classification problem, i.e., each pixel is classified as the myocardium or the background. This is a class imbalance problem, since there is a lower percentage of pixels in the CMR image that corresponds to the myocardium class. So, suppose that a model simply predicts all pixels to be belonging to the background, its accuracy performance will still be quite good even though it fails to actually detect any pixels belonging to the myocardium. Hence, a new accuracy function based on the dice metric (refer to Equation 1 in Section 3.2) is designed as a precision indicator of the image segmentation, to be used during the network training.

\section{Experiment}

\subsection{Image set}

The CMR dataset used in our study is from York University [19]. The dataset contains 5,011 myocardiumannotated CMR frames acquired from 33 subjects. Among them, 3,229 frames from 21 subjects were for network training, 861 frames from the other 6 subjects were for validation, and 921 frames from the remaining 6 subjects were for testing. We perform affine transformations (rotation and flipping) to augment the training set in an effort to improve the model generalization.

\subsection{Evaluation metric}

Dice metric (DM) is the most popularly used metrics to perform a quantitative evaluation of the segmentation results. We evaluated the performance of our deep convolutional network by comparing the segmented areas with the manually segmented ones in terms of DM. Let $A$ represent the area formed by the automatically segmented contour and $G$ represent the area formed by the manually segmented contour, then DM can be defined by

$$
D M=\frac{2(A \cap G)}{A+G}
$$


where $A \cap G$ denotes the intersection area between $A$ and $G$, and $A+G$ denotes the union of $A$ and $G$. The value of DM is always between 0 and 1 , with higher value indicating better match between the automatically segmented area and the corresponding manually segmented ones.

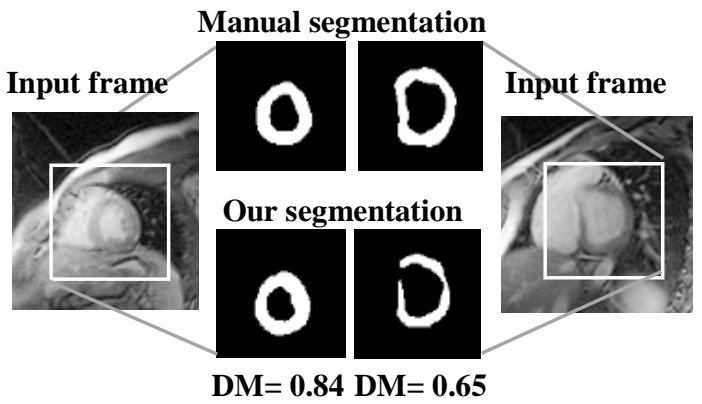

Figure 2. Two typical segmentation results: good segmentation with $\mathrm{DM}=0.84$ (representing most of test frames) and a broken segmentation with $\mathrm{DM}=0.65$

Table.2 The performance of the proposed model on Subject\#05 from the test set. Due to space constraint, only results at every 10 frames are shown here.

\begin{tabular}{|l|l|}
\hline ID & DM \\
\hline Frame \#001 & 0.81 \\
\hline Frame \#011 & 0.82 \\
\hline Frame \#021 & 0.83 \\
\hline Frame \#031 & 0.83 \\
\hline Frame \#041 & 0.79 \\
\hline Frame \#051 & 0.83 \\
\hline Frame \#061 & 0.71 \\
\hline Frame \#071 & 0.80 \\
\hline Frame \#081 & 0.76 \\
\hline Frame \#091 & 0.80 \\
\hline Frame \#101 & 0.83 \\
\hline Frame \#111 & 0.86 \\
\hline Frame \#121 & 0.83 \\
\hline Frame \#131 & 0.73 \\
\hline Frame \#141 & 0.48 \\
\hline Frame \#151 & 0.64 \\
\hline Summary & 0.78 \\
\hline Average & 0.07 \\
\hline Stdev & 0.48 \\
\hline Min & 0.86 \\
\hline Max
\end{tabular}

\subsection{Testing results}

The trained deep learning model is used to segment the LV myocardium on all 154 short-axis frames of Subject \#05 from the test set. Table 2 lists the DM values at every 10 frames due to space limitation. The DM values are calculated by using Eqn. (1). Note that the summary results are based on all 154 frames and not just the frames shown in the table. It can be seen that most of the segmented results are reasonable since their associated $\mathrm{DM}$ values are above 0.80 . The cases with low DM values are due to the partially segmented myocardium region. Two examples of segmentation results are shown in Figure 2. Overall, the DM value of Subject \#05 is $0.78 \pm 0.07$.

Table 3. Evaluation results of trained FCN in terms of DM values for all 6 subjects from the test set.

\begin{tabular}{|l|l|}
\hline ID & DM \\
\hline Subject \#05 & 0.78 \\
\hline Subject \#10 & 0.68 \\
\hline Subject \#18 & 0.79 \\
\hline Subject \#22 & 0.74 \\
\hline Subject \#27 & 0.72 \\
\hline Subject \#32 & 0.77 \\
\hline Summary \\
\hline Average & 0.75 \\
\hline Stdev & 0.04 \\
\hline Min & 0.68 \\
\hline Max & 0.79 \\
\hline
\end{tabular}

The trained deep learning model is further tested on all 6 subjects in the test set, with a total of 921 frames. The segmentation results from our method are compared to manually segmented contours by expert cardiologists. These ground truths for the segmentation are available on the York University website [19]. Table 3 summarizes the statistics of the evaluation measures on each of the 6 subjects. The overall result by the proposed model is $0.75 \pm 0.04$, which is promising considering that it is purely obtained from a deep learning approach. It is noted that our result still has a gap with the best published myocardium segmentation result of $\mathrm{DM}=0.84$. This was achieved by the state-of-the-art semi-automatic segmentation method [20] on the SATCOM 2011 Challenge Data [21], in which an expert guide was employed to improve the segmentation results.

\section{Conclusion}

Cardiac image segmentation plays a crucial role and allows for a wide range of applications, including quantification of volume, computer-aided diagnosis, localization of pathology, and image-guided 
interventions. We had proposed a framework based on deep convolutional neural networks for a fully automatic segmentation of the $\mathrm{LV}$ myocardium from short-axis CMR image sequences. In its current form, the proposed one-step deep learning method is unable to match the results of the state-of-the-art myocardium segmentation methods. Nevertheless, it delivers a promising first-pass segmentation results. Moving ahead, we aim to develop a hybrid method by fine-tuning the deep neural network, augmenting with more training samples, and postprocessing broken myocardium segmentations.

\section{References}

[1] Y. Bengio, A. Courville, P. Vincent. Representation learning: A review and new perspectives. IEEE Trans. On Pattern Analysis and Machine Intelligence 2013:35-8:1798-2828.

[2] Y. LeCun, Y. Bengio, G. Hinton. Deep learning. Nature 2015;521:436-444.

[3] J. Schmidhuber. Deep learning in neural networks: An overview. Neural Networks 2015;61:85-117.

[4] M.R. Avendi, Arash Kheradvar, Hamid Jafarkhani. A combined deep-learning and deformable-model approach to fully automatic segmentation of the left ventricle in cardiac MRI. Medical Image Analysis 2016;30:108-119.

[5] O. Emad, I.A. Yassine, A.S. Fahmy. Automatic localization of the left ventricle in cardiac MRI images using deep learning. IEEE Engineering in Medicine and Biology Society 2015:683-686.

[6] T.A. Ngo, G. Carneiro. Left ventricle segmentation from cardiac MRI combining level set methods with deep belief networks. IEEE International Conference on Image Processing 2013:695-699.

[7] H. Hu, Z. Gao, L. Liu, H. Liu, J. Gao, S. Xu, W. Li, L. Huang. Automatic Segmentation of the Left Ventricle in Cardiac MRI Using Local Binary Fitting Model and Dynamic Programming Techniques. PLoS ONE 2014;9-12:1-17.

[8] M.G. Uzunbaş, S. Zhang, K.M. Pohl, D. Metaxas, L. Axel. Segmentation of myocardium using deformable regions and graph cuts. IEEE International Symposium on Biomedical Imaging 2012:254-257.

[9] P. Radau, Y. Lu, K. Connelly, G. Paul, A.J. Dick, G.A. Wright. Evaluation framework for algorithms segmenting short axis cardiac MRI. MIDAS J. Cardiac MR Left Ventricle Segmentation Challenge 2009.

[10] J. Sjögren, J.F.A. Ubachs, H. Engblom, M. Carlsson, H. Arheden, E. Heiberg. Semi-automatic segmentation of myocardium at risk in T2-weighted cardiovascular magnetic resonance. Journal of Cardiovascular Magnetic Resonance 2012;14-1:1-
11.

[11] J. Tufvesson, M. Carlsson, A.H. Aletras, H. Engblom, J-F. Deux, S. Koul, P. Sörensson, J. Pernow, D. Atar, D. Erlinge, H. Arheden, E. Heiberg. Automatic segmentation of myocardium at risk from contrast enhanced SSFP CMR: validation against expert readers and SPECT. BMC Med Imaging 2016;16-1:19.

[12] C. Petitjean, J-N. Dacher. A review of segmentation methods in short axis cardiac MR images. Medical Image Analysis 2011;15-2:169-184.

[13] Y. LeCun, L. Bottou, Y. Bengio, P. Haffner. Gradient-based learning applied to document recognition, Proceedings of the IEEE 1998;8611:2278-2324.

[14] K. Simonyan, A. Zisserman. Very Deep Convolutional Networks for Large-Scale Image Recognition. arXiv preprint arXiv:1409.1556 2014.

[15] C. Szegedy, W. Liu, Y. Jia, P. Sermanet, S. Reed, D. Anguelov, D. Erhan, V. Vanhoucke, A. Rabinovich. Going deeper with convolutions. arXiv:1409.4842 2014.

[16] K. He, X. Zhang, S. Ren, J. Sun. Deep residual learning for image recognition. arXiv:1512.03385 2015.

[17] J. Long, E. Shelhamer, T. Darrell. Fully convolutional networks for semantic segmentation. arXiv:1411.4038 2015.

[18] L.C. Chen, G. Papandreou, I. Kokkinos, K. Murphy, A. L. Yuille. Semantic image segmentation with deep convolutional nets and fully connected CRFs. arXiv:1412.7062 2014.

[19] A. Andreopoulos, J.K. Tsotsos. Efficient and Generalizable Statistical Models of Shape and Appearance for Analysis of Cardiac MRI. Medical Image Analysis 2008;12-3:335-357.

[20] B. Li, Y. Liu, C.J. Occleshaw, B.R. Cowan, A.A. Young. In-line automated tracking for ventricular function with magnetic resonance imaging. Journal of American College of Cardiology: Cardiovascular Imaging 2010;3-8:860-866.

[21] A. Suinesiaputra, B.R. Cowan, J.P. Finn, C.G. Fonseca, A.H. Kadish, D.C. Lee, P. MedranoGracia, S.K. Warfield, W. Tao, A.A. Young. Left ventricular segmentation challenge from cardiac MRI: a collation study. Statistical Atlases and Computational Models of the Heart, Imaging and Modelling Challenges 2012:88-97.

Address for correspondence.

$\mathrm{X}$. Yang

Institute of High Performance Computing

1 Fusionopolis Way, Connexis (North) \#16-16

Singapore 138632

yangx@ihpc.a-star.edu.sg 